\title{
Development of Optical Diagnostic Techniques to Correlate Mixing and Auto-lgnition Processes in High Pressure Diesel Jets
}

\author{
Gilles Bruneaux \\ Institut français du pétrole, IFP, Division Techniques d'Applications Énergétiques, 1-4 avenue de Bois-Préau, 92852 Rueil-Malmaison Cedex - France \\ e-mail: gilles.bruneaux@ifp.fr
}

\begin{abstract}
Résumé - Développement de techniques de diagnostic optique pour corréler le mélange et l'autoinflammation en injection directe Diesel haute pression - Une technique de visualisation du carburant par Fluorescence Induite par Laser (LIF) en présence d'oxygène est développée pour l'étude de l'injection directe Diesel haute pression. Un nouveau traceur, le 5 nonanone, est sélectionné sur la base d'une étude bibliographique. Des essais sont effectués avec ce dernier dans une enceinte haute pression haute température qui reproduit les conditions thermodynamiques rencontrées dans la chambre de combustion d'un moteur Diesel au moment de l'injection. Ils montrent que le signal de fluorescence collecté à l'air est suffisamment important pour permettre d'envisager des visualisations du carburant en présence d'oxygène dans des configurations difficiles, comme par exemple par illumination globale. Ensuite, la technique de LIF à l'air est combinée à une visualisation rapide de la chimiluminescence afin d'analyser la corrélation entre la structure du mélange et l'auto-inflammation du jet Diesel. Dans la configuration d'un jet libre et dans la gamme de paramètres étudiés, l'auto-inflammation est principalement localisée dans des zones riches en amont de la zone de mélange. Dans la configuration d'un jet interagissant avec une paroi perpendiculaire, l'auto-inflammation apparaît dans le centre du jet et se propage aux extrémités au voisinage de la paroi.
\end{abstract}

\footnotetext{
Abstract - Development of Optical Diagnostic Techniques to Correlate Mixing and Auto-Ignition Processes in High Pressure Diesel Jets - A tracer laser-induced fluorescence (LIF) technique for the visualization of fuel distribution in the presence of oxygen was developed and then used sequentially with high speed chemiluminescence imaging to study the correlation between the mixing and auto-ignition processes of high pressure Diesel jets. A single hole common rail Diesel injector allowing high injection pressures up to $150 \mathrm{MPa}$ was used. The reacting fuel spray was observed in a high pressure, high temperature cell that reproduces the thermodynamic conditions which exist in the combustion chamber of a Diesel engine during injection. Both free jet and flat wall impinging jet configurations were studied. Several tracers were first considered with the objective of developing a tracer-LIF technique in the presence of oxygen. 5-nonanone was selected for its higher fluorescence efficiency. This technique was subsequently combined with high speed chemiluminescence imaging to study the correlation between mixing and auto-ignition. In the free jet configuration and for the parameter range studied, it was found that auto-ignition is preferentially located in rich regions of the upstream mixing zone. Also, in the jet wall configuration, auto-ignition appears in the centre of the jet and propagates towards the periphery, in the vicinity of the wall.
} 


\section{DEFINITIONS, ACRONYMS, ABBREVIATIONS}

$d_{i} \quad$ Nozzle hole diameter $(\mathrm{mm})$

$d_{n w}$ Distance between the nozzle and the wall in the jet wall configuration ( $\mathrm{mm}$ )

LIF Laser Induced Fluorescence

LIEF Laser Induced Exciplex Fluorescence

$P_{i n j} \quad$ Injection pressure (bar)

$t_{i} \quad$ Time between the start of injection and imaging (ms)

$x \quad$ Horizontal abscissa, origin at the nozzle hole centre $(\mathrm{mm})$

$y \quad$ Vertical abscissa, origin at the nozzle hole centre $(\mathrm{mm})$

\section{INTRODUCTION}

High Pressure Direct Injection (HPDI) is now a well-established technology which allows the efficient reduction of engine-out emissions in DI Diesel engines. But although ongoing research on HPDI has led to an improved understanding of the physical processes occurring in the combustion chamber, further studies are required in order to ensure that this technology is exploited to its full potential in terms of optimizing combustion processes. Numerous studies on the mixing [1-5] and combustion processes [6-8] have been reported in the literature, however there is a need for an improved understanding of the correlation between these two processes. This is the primary aim of the present work and is based upon the application of optical diagnostics.

In order to achieve this goal, sequential diagnostics of both mixing and combustion processes are to be applied to a Diesel jet. However, established diagnostics for mixing studies such as Laser Induced Fluorescence (LIF) suffer from significant quenching due to the presence of oxygen molecules. This effect explains why most mixing studies with LIF are performed in an oxygen-free environment, which is inevitably a major limitation when mixing has to be observed under reacting (i.e. combustion) conditions. A tracer LIF technique enabling mixing visualizations in the presence of oxygen therefore needs to be developed. This is the second aim of the present work.

The first part of the investigation was then to select a suitable LIF tracer which enables visualization of the fuel mixture in the presence of oxygen. In order to achieve this, several fluorescent tracers suitable for a Diesel type fuel were considered and tested. This particular LIF technique was then combined with high speed chemiluminescence imaging of the combustion process. Indeed, previous work [6] has shown that chemiluminescence imaging is a useful technique for visualization of auto-ignition sites since, during the first stages of combustion, the chemiluminescence emission correlates spatially with the location of auto-ignition precursors (formaldehyde). Visualizations of the mixture structure and auto-ignition sites were performed during a single cycle (i.e. single injection event), enabling a detailed analysis of the correlation between the two processes. Single cycle imaging using both techniques is necessary for unambiguous interpretation of the data. Indeed, simply taking into account the mean averaged images can be misleading since the mean averaged images are not necessarily representative of the single-shot image [4].

A high pressure cell was used in the present study since it provides extensive optical access for the application of laser diagnostics. This experimental apparatus is therefore firstly presented, followed by the experimental techniques. The results section first of all discusses the selection of the fluorescent tracer and subsequently the studies of the correlation between fuel mixture distribution and auto-ignition, for both the free jet and wall jet configurations.

\section{EXPERIMENTAL APPARATUS}

\subsection{High Pressure Cell}

The results presented here have been performed in a highpressure, high-temperature cell which enables the study of spray behaviour under thermodynamic conditions similar to those of a Diesel engine at a specified injection timing. The major advantage of performing measurements in an optical cell is that the gas phase conditions such as air flow, temperature and pressure are more easily controlled than in an engine, whilst providing better optical access for the application of laser diagnostic techniques. The flow in the cell is essentially that induced by the spray itself.

Further details concerning the cell and its operation can be found in previous publications [9]. The injector is mounted within the top face of the cell. Five $80 \mathrm{~mm}$ diameter $20 \mathrm{~mm}$ thick sapphire windows can be mounted on the other remaining faces.

Prior to performing the experiments, the gases within the cell are heated and pressurized to reproduce typical in-engine conditions by a pre-combustion technique similar to that described in [10]. The start of injection (SOI) takes place at a specified time after pre-combustion ignition. The SOI time is determined so that injection takes place when the temperature and pressure in the cell have reached the desired values. The pre-combustion gas mixture can be computed to consume a certain quantity of the oxygen initially introduced in the cell. As a result, experiments can be performed at various oxygen concentrations. For the experiments presented in this paper, "oxygen-free" corresponds to $0 \%$ oxygen during injection while "in the presence of oxygen" corresponds to $21 \%$ oxygen by volume in the surrounding gases. An ambient density of $25 \mathrm{~kg} / \mathrm{m}^{3}$ and ambient temperature of $800 \mathrm{~K}$ were used for all the tests reported in this paper. 


\subsection{Wall Holder}

In order to study jet wall impingement, a flat metal insert (also referred to as a wall) was mounted in the centre of the cell. The wall holder was configured so that the metal insert plane was perpendicular to the jet axis. The distance $d_{n w}$ between the nozzle hole and the wall was adjustable between 21.5 and $35.0 \mathrm{~mm}$. This range is characteristic of the distance between the injector nozzle and the piston bowl wall of light duty Diesel engines. For the experiments presented in the paper, distances of 25 and $35 \mathrm{~mm}$ were used.

Also, since the wall is heated by the pre-combustion event, during fuel injection the surface wall temperature is expected to be higher than its initial temperature before precombustion (i.e. $454 \mathrm{~K}$ ), but it was not measured during this study. However, measurements made in similar conditions elsewhere [11] with thermocouples reported surface wall temperatures of $500 \mathrm{~K}$.

More detailed information on the wall holder and its effect on chamber conditions are presented in [5].

\subsection{Injection System}

The injector and its driver electronics were supplied by CRT AG. A single-hole nozzle with nominal hole diameter/length of 0.15/0.6 mm was used. A hydro-pneumatic pump manufactured by Top Industrie SA was used to supply fuel at a constant injection pressure. The injection duration for all tests presented in the paper is $2 \mathrm{~ms}$. Results of mass flow rate measurement with this injector are available in [4].

\subsection{Tracer LIF Selection}

\subsubsection{Principle}

The fluorescent tracer commonly used to visualize the mixture formation of Diesel injection is TMPD. It is principally selected since its boiling temperature of $260^{\circ} \mathrm{C}$ at ambient conditions is representative of the middle boiling point behaviour of standard Diesel fuels, and also because it enables a distinction between the liquid and vapour phases through the formation of an Exciplex (more information on the Exciplex formation between TMPD and $\alpha$ methylnaphtalene can be found in [2]). However, TMPD and TMPD/ $\alpha$-methylnaphtalene exciplex fluorescence for visualization in the vapour and liquid phases respectively are significantly quenched by oxygen molecules [2, 12]. The resulting reduction of signal to noise ratio makes mixture visualization in the presence of oxygen extremely challenging. Other parameters such as temperature, pressure, gas composition can influence the fluorescence signal but oxygen quenching is the most limiting in term of signal to noise ratio. The tracer TMPD has been evaluated within the present study, whilst in addition, other tracers were investigated which are less sensitive to oxygen quenching and might therefore result in improved LIF signal to noise ratio. In general, ketone molecules are good candidates since photo-physical phenomena occurring during fluorescence excitation for this family of molecules makes them less sensitive to quenching [13]. Another important criterion for the choice of fluorescent tracer is that the boiling temperature should be similar to that of the fuel. Applying these criteria, two molecules were identified: 5-nonanone with a boiling temperature of $188^{\circ} \mathrm{C}$, and 2-decanone with a boiling temperature of $210^{\circ} \mathrm{C}$. Previous tests with 5-nonanone [14] show that a good signal to noise ratio can be obtained in the presence of oxygen. However, tracer decomposition in the fuel supply system might lead to experimental difficulties.

The fuels used for the tests differ according to the tracer properties. When TMPD, excited at $355 \mathrm{~nm}$, is used as a tracer, the base fuel was a mixture of $70 \% n$-decane (boiling point $174.1^{\circ} \mathrm{C}$ ) with $30 \% \alpha$-methyl-naphthalene (boiling point $244.8^{\circ} \mathrm{C}$ ) by volume. This base fuel was then doped with $0.5 \%$ of TMPD by mass. This mixture has been used in previous experiments $[4,5]$. In contrast however, the absorption bands of 5-nonanone and 2-decanone are in the range 220-270 $\mathrm{nm}$ and are therefore excited at $266 \mathrm{~nm}$ by the fourth harmonic of a Nd:YAG laser. Since $\alpha$-methyl-naphthalene also emits fluorescence upon excitation at $266 \mathrm{~nm}$ [12], significant LIF signal interference occurs and it was thus necessary to select other non-fluorescing base fuels (or fuel mixtures). $n$-dodecane was chosen as the base fuel since it does not fluoresce when excited at $266 \mathrm{~nm}$ and its boiling point of $216^{\circ} \mathrm{C}$ at ambient conditions is representative of the boiling point of the more volatile components within standard Diesel fuels. $1 \%$ tracer (either 5-nonanone or 2-decanone) by mass was then added to the base fuel. The properties of the three tracers and base fuels are summarized in Table 1.

TABLE 1

Summary of tracers and base fuels properties

\begin{tabular}{l|l|l|l|l}
\hline Tracer & $\begin{array}{l}\text { Boiling } \\
\text { point } \\
\left({ }^{\circ} \mathrm{C}\right)\end{array}$ & $\begin{array}{l}\text { Excitation } \\
\text { wavelength } \\
(\mathrm{nm})\end{array}$ & Base fuel & $\begin{array}{l}\text { Boiling point } \\
\left({ }^{\circ} \mathrm{C}\right)\end{array}$ \\
\hline TMPD & 260 & 355 & $\begin{array}{l}70 \% \text {-decane } / \\
30 \% \alpha \mathrm{MN}\end{array}$ & $174.1 / 244.8$ \\
\hline 5-nonanone & 188 & 266 & $n$-dodecane & 216 \\
\hline 2-decanone & 210 & 266 & $n$-dodecane & 216 \\
\hline
\end{tabular}

An important factor that should be noted are the differences in cetane number $(\mathrm{CN})$ between the two base fuels used in this study. The overall $\mathrm{CN}$ of the $n$-decane $(\mathrm{CN}$ $74) / \alpha$-methylnaphthalene ( $\mathrm{CN} 0$ ) mixture is 53, comparable to a standard Diesel fuel whilst the $\mathrm{CN}$ of $n$-dodecane is 80 . As a result, the auto-ignition delays would be 
expected to be shorter in the case of the $n$-dodecane base fuel. These differences do not affect the first part of the study which is dedicated to an analysis in terms of the tracer LIF signal characteristics and propensity to $\mathrm{O}_{2}$ quenching, since the primary focus here is on the fuel mixture distribution and therefore prior to auto-ignition/combustion.

\subsubsection{Influence of the Degree of Optical Access}

Although one of the first objectives was to assess the potential of fluorescent tracers suitable for experiments in reacting (i.e. in the presence of oxygen) conditions to investigate the correlation between mixture distribution and combustion, there are wider implications. The selection of such a tracer might prove to be useful for optical engine applications. Fuel visualizations in optically accessible engines are often performed in oxygen-free environments due to the problems of LIF tracer signal quenching, however, under such 'non-firing' conditions, the combustion chamber wall and bulk gas temperatures are not representative. Fuel visualization by tracer LIF in the presence of oxygen would therefore be of great interest since it would allow measurements to be performed under more representative engine operating conditions. However, the image signal to noise ratio for those tests performed in the high pressure cell might not be comparable to in-cylinder engine visualizations since the optical access in the latter case is significantly reduced. As a result, in order to evaluate tracer LIF signal to noise ratios in both the cell and engine conditions, experiments were performed on the high temperature/pressure cell with the objective of replicating the comparatively limited optical access that is typically available on optical engines. More details of the set-up are provided in the following subsection.

\subsubsection{Experimental Setup}

The experimental setup for LIF tracer selection is presented in Figure 1. Tracer fluorescence was excited by a frequencytripled Nd:YAG laser at $355 \mathrm{~nm}$ in the case of TMPD and with a frequency-quadrupled Nd:YAG laser at $266 \mathrm{~nm}$ for the 5-nonanone and 2-decanone tracers. A cylindrical lens $(f=100 \mathrm{~mm})$ was coupled to a spherical lens $(f=1000 \mathrm{~mm})$ to produce a laser sheet which entered the cell through one of the side windows. Sapphire windows were used for $355 \mathrm{~nm}$ excitation and quartz in the case of $266 \mathrm{~nm}$ excitation. To avoid possible data misinterpretation, part of the laser sheet was masked so as to avoid illuminating the liquid phase of the spray. The tracer fluorescence signal was collected by two 16 bit intensified CCD cameras (Princeton Instruments PIMAX) through two diametrically opposed windows located perpendicularly to the laser sheet. One of the cameras hereafter referred to as camera A was located at $48 \mathrm{~mm}$ from the centre of the cell and was coupled to a $45 \mathrm{~mm} \mathrm{f} / 1.8 \mathrm{UV}$ lens. This camera set-up corresponds to optimum conditions for light collection as a result of the extensive optical access provided by the test cell. The other camera hereafter referred to as camera B was located at $60 \mathrm{~mm}$ from the centre of the cell and was coupled to a $105 \mathrm{~mm}, f / 4.5 \mathrm{UV}$ lens. Moreover a mask which provided a $4 \mathrm{~cm}$ diameter circular aperture was placed between the cell window and the camera. This configuration was intended to represent typical conditions encountered in optical engine experiments in which image detection is more severely limited due to a combination of the effects of reduced optical access and the greater distance between the object (i.e. plane illuminated by the laser sheet) and image (i.e. CCD detector location) plane. Using two cameras at the same time was not mandatory for the comparison of the two configurations since it could have been made in sequential measurements, but it clearly facilitates the process.

Specific characteristics of the setup are required depending on the fluorescence tracer used and these are discussed below.

For LIF experiments with TMPD, the laser energy measured at the laser outlet at $355 \mathrm{~nm}$ was $280 \mathrm{~mJ}$, so that the $6 \times 0.1 \mathrm{~cm}^{2}$ laser sheet formed after passing through the lenses had a power intensity of $1.6 \times 10^{7} \mathrm{~W} / \mathrm{cm}^{2}$. Band pass interference filters $400 \mathrm{~nm}$ (FWHM $25 \mathrm{~nm}$ ) and $401 \mathrm{~mm}$ (FWHM $10 \mathrm{~nm}$ ) were placed respectively between the cell and cameras A and B. These filters are used to detect the fluorescence signal corresponding to the TMPD molecule in the vapour phase [4].

For LIF experiments with 5-nonanone and 2-decanone, a laser beam energy of $80 \mathrm{~mJ}$ was measured at the laser outlet at $266 \mathrm{~nm}$, so that the $4.5 \times 0.1 \mathrm{~cm}^{2}$ laser sheet formed after passing through the lenses has a power intensity of $310^{6} \mathrm{~W} / \mathrm{cm}^{2}$. Two identical band pass interference filters

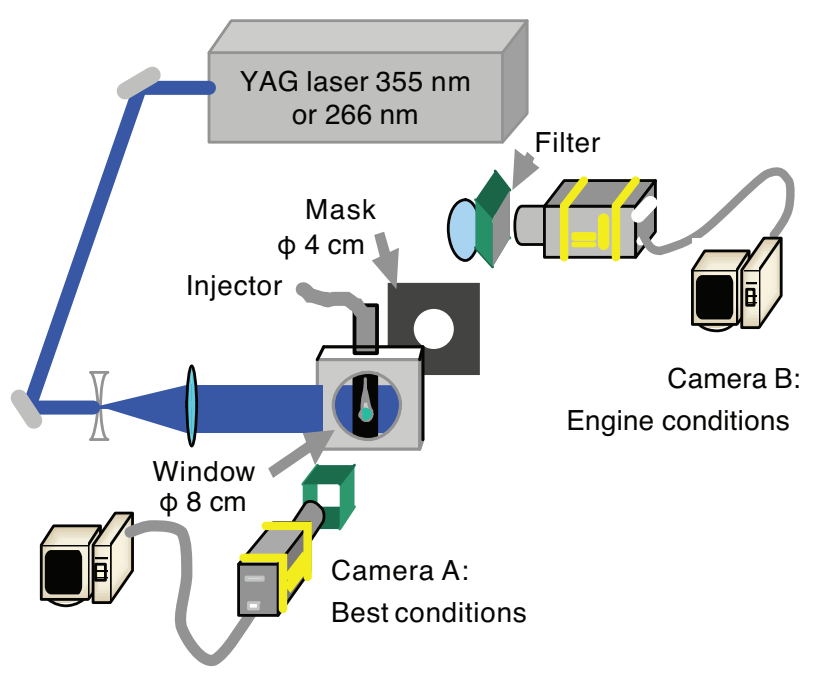

Figure 1

Experimental setup for tracer LIF selection. 
$327 \mathrm{~nm}$ (FWHM $75 \mathrm{~nm}$ ) were located between the cell and cameras A and B for detection of the fluorescence signal from the tracer molecules.

\subsection{High Speed Tracer Lif and Combustion Visualizations}

\subsubsection{Principle}

The principle of high speed tracer LIF and combustion visualization is to combine two diagnostics giving information on mixing and auto-ignition in the same event by fast imaging. Concerning auto-ignition, intensified direct visualization of natural combustion light was used because it was shown in previous work $[7,15,16]$ that the localization of the light collected by direct visualization corresponds the localization of auto-ignition, the latter being characterized by the presence of formaldehyde during the first reactions of fuel decomposition.

\subsubsection{Experimental Setup}

The experimental setup for high speed tracer LIF and combustion visualizations is presented in Figure 2. The laser diagnostic set-up used here was identical to that used in the 5-nonanone experiments discussed above. However, the fluorescence and combustion signals were collected with an intensified high speed Lavision Ultraspeedstar camera. The exposure time of all frames of the camera was set to $5 \mu \mathrm{s}$. With this setting, a satisfactory compromise was obtained between the tracer LIF and combustion image intensities. A set of High Pass $(320 \mathrm{~nm})$ and Low Pass (400 nm) optical filters were located between the cell and the camera for fluorescence signal detection. This combination of filters was selected following the 5-nonanone tests in order to further optimize signal to noise ratio. It is also compatible with the chemiluminescence emission spectra.

\subsubsection{Synchronization Method}

Sequential tracer LIF and combustion (chemiluminescence) imaging was achieved (in the same event) with the high speed Lavision Ultraspeedstar camera. This camera is capable of acquiring up to 16 consecutive images at very short intervals: down to 20 to $50 \mu$ s for this experiment. The camera was adjusted to observe the evolution of chemiluminescence evolution except for one image (the first one) for which the camera aperture was synchronized with the laser pulse so that the LIF signal was collected in addition to the chemiluminescence signal, as shown in Figure 3. If the laser pulse is synchronized at a timing where chemiluminescence signal is negligible, all the collected signal at that timing originates from LIF. The resulting image sequence therefore contains one LIF image followed by the evolution of the chemiluminescence signal.

\subsubsection{Image Post Processing}

For a better interpretation and analysis of the results and in particular the correlation between mixture distribution and combustion, the sequence of LIF and chemiluminescence images corresponding to each event was post processed in order to superpose the information on mixing and auto-ignition on a single image. The post processing method is presented in Figure 4. Firstly, contour detection was applied to

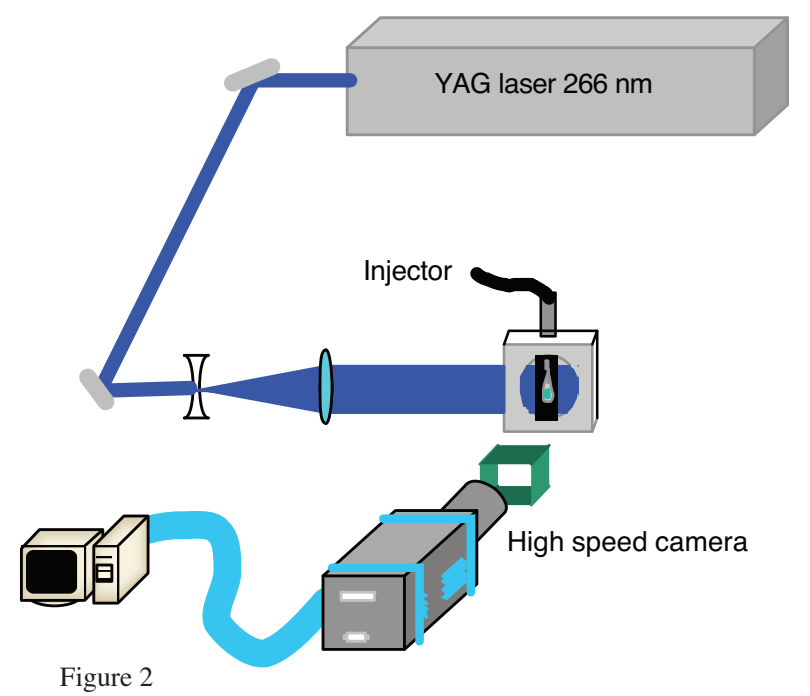

Figure 2

Experimental setup schematic for high speed tracer LIF and combustion visualizations.

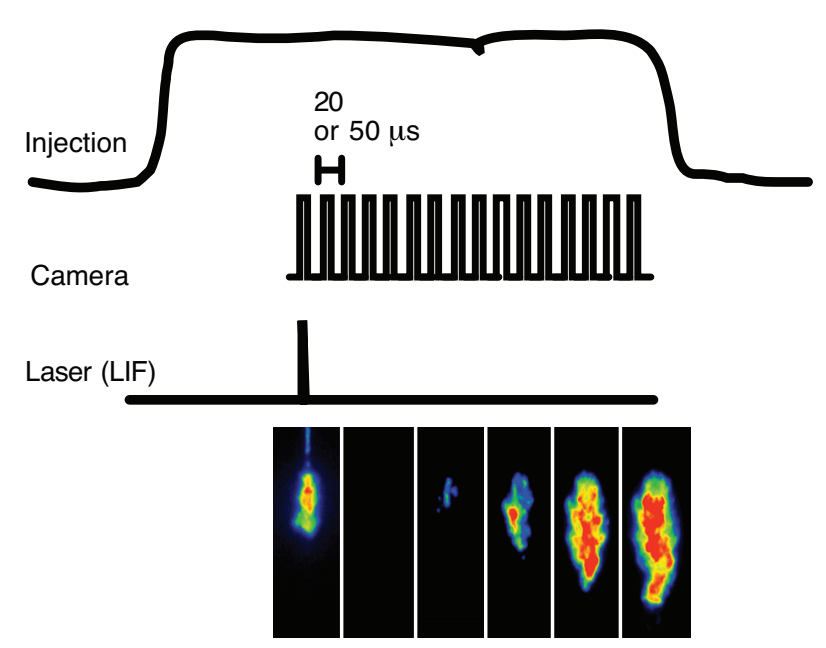

Figure 3

Illustration of synchronization method. 


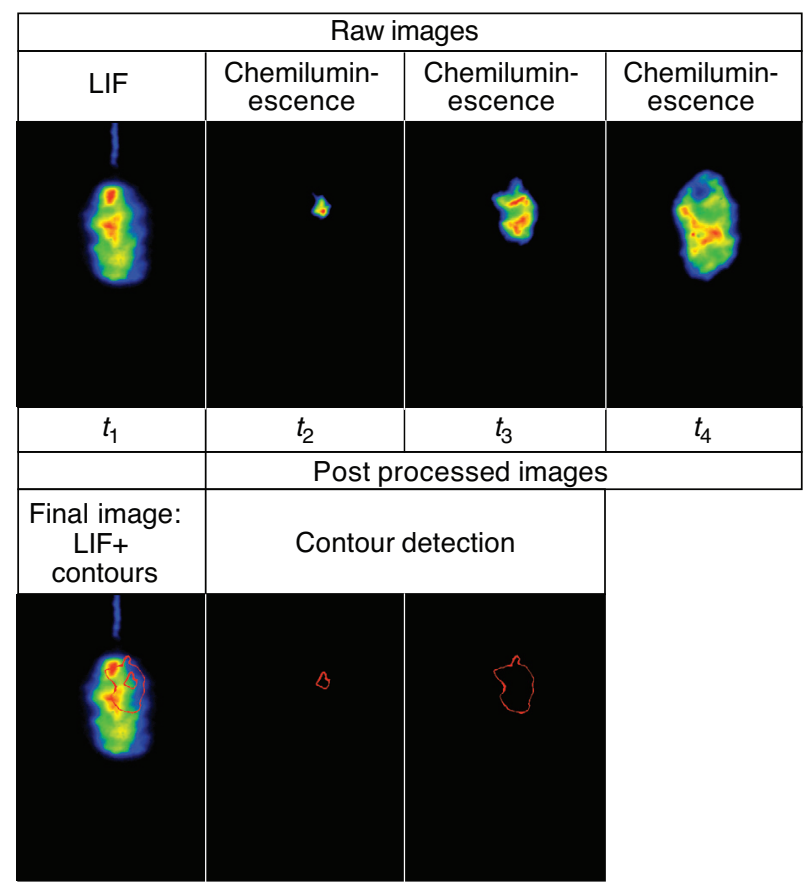

Figure 4

Post processing principle of high speed camera images: contour detection of chemiluminescence images (autoignition site location) and superposition on LIF image.

the first two chemiluminescence images in order to obtain the spatial location of auto-ignition sites. Then the corresponding contours were superposed on the LIF image. The contours were determined using a threshold method, where a fixed threshold level was set visually. The resulting image gathers information on the mixture, through the tracer fluorescence field, and on the location of auto-ignition that follows, through the contours of the first chemiluminescence images. Comparing the relative position of the two contours also provides information on the evolution of auto-ignition. Each post processed image corresponds to three timings: $t_{1}$ is the timing of the LIF image, $t_{2}$ is the timing of the first contour and $t_{3}$ is the timing of the second contour.

\section{RESULTS}

\subsection{Tracer LIF Selection}

\subsubsection{TMPD}

The effect of the presence of oxygen on TMPD fluorescence was investigated. The fluorescence signal was collected in the vapour part of a Diesel jet, in conditions where all the parameters are comparable, except for the oxygen concentra- tion within the ambient gases. The collection parameters such as camera gain were also identical so that the images provide a direct indication of the quantum efficiency of the fluorescence in the different environments. Mean averaged images obtained from 3 individual images are shown in Figure 5.

The fluorescence signal is strongly reduced by oxygen: the signal collected in the centre of the jet is approximately 4 times lower in the presence of oxygen. This result is due to the quenching of fluorescence by oxygen molecules, as discussed before.

For further investigation, TMPD LIF images in the presence of oxygen were acquired with maximum camera gain. The latter was required in order to compensate for the signal reduction in the presence of oxygen. Typical examples of resulting single shot images obtained with the two cameras are compared in Figure 6. The two images are acquired simultaneously through the two opposite windows and therefore they reveal a very similar structure. The signal intensity of the image obtained using camera B is clearly reduced compared to that acquired by camera $\mathrm{A}$. This is due to the lower signal collection efficiency of the setup of camera B which is intended to reproduce a typical engine setup. Approximately four times less signal is collected with camera $\mathrm{B}$, under engine-like conditions, compared to camera A.

The images of Figure 6 show that although TMPD fluorescence signal intensity is strongly reduced by oxygen quenching, it is still possible to visualize fuel concentration

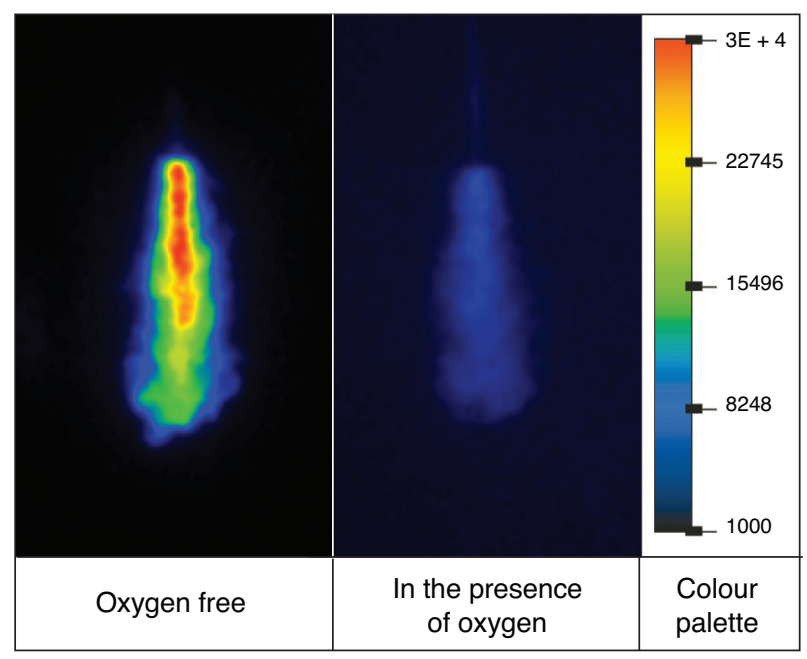

Figure 5

Comparison of mean-average ( 3 individual) TMPD LIF images obtained with and without oxygen. Camera A (cell conditions), relative gain $4.2, P_{i n j}=1200 \mathrm{bar}, t_{i}=0.8 \mathrm{~ms}$, image size $40 \times 61 \mathrm{~mm}^{2}$. 
by LIF in the presence of oxygen when optimum conditions for signal collection are available. For the setup representing optical engine conditions, the signal to noise ratio is low and it appears difficult to perform satisfactory visualizations under such conditions.

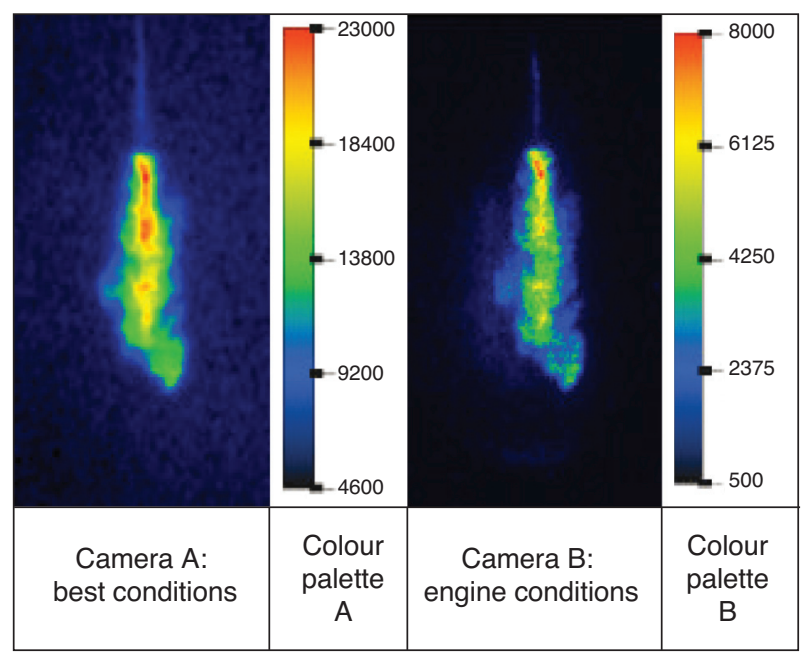

Figure 6

Comparison of single shot TMPD LIF images obtained with camera $\mathrm{A}$ and $\mathrm{B}$ (cell and engine conditions) in the presence of oxygen. Relative gain $13, P_{i n j}=1200$ bar, $t_{i}=0.8 \mathrm{~ms}$, image size $40 \times 61 \mathrm{~mm}^{2}$.
A more in-depth analysis of the effects of oxygen on TMPD LIF measurements was performed. Indeed, the fluorescence signal in an oxygen free environment is at first order proportional to the tracer concentration, and therefore to the fuel concentration [4]. Since the presence of oxygen significantly affects the fluorescence intensity through quenching, a detailed analysis of the differences between the two signals can provide information on the oxygen distribution in the jet. This phenomena was already applied to gasoline engine investigations to directly measure the air fuel ratio [17], although recent work on toluene [18] showed that other fast photo-physical phenomenon could compensate quenching at high temperature, limiting the accuracy of the technique.

Figure 7 shows TMPD fluorescence intensity profiles obtained with and without oxygen. The two sets of corresponding images were acquired with different camera gains in order to allow good imaging conditions for both cases. This implies that the comparison of absolute signal intensities is not possible. Indeed, the purpose of this figure is to compare the relative spatial distributions of signal intensity. The profiles were extracted from mean averaged images (10 images) along the injector axis and perpendicular to it. The absolute signal intensities of the TMPD LIF images obtained in the presence of oxygen were significantly lower than those obtained in an oxygen free environment. Therefore, in order to compare the spatial distribution for the two cases, it was necessary to shift the image intensities to a similar level to facilitate comparison of the data. After doing so, the spatial distribution of the fluorescence intensity profiles obtained in an oxygen free environment and in
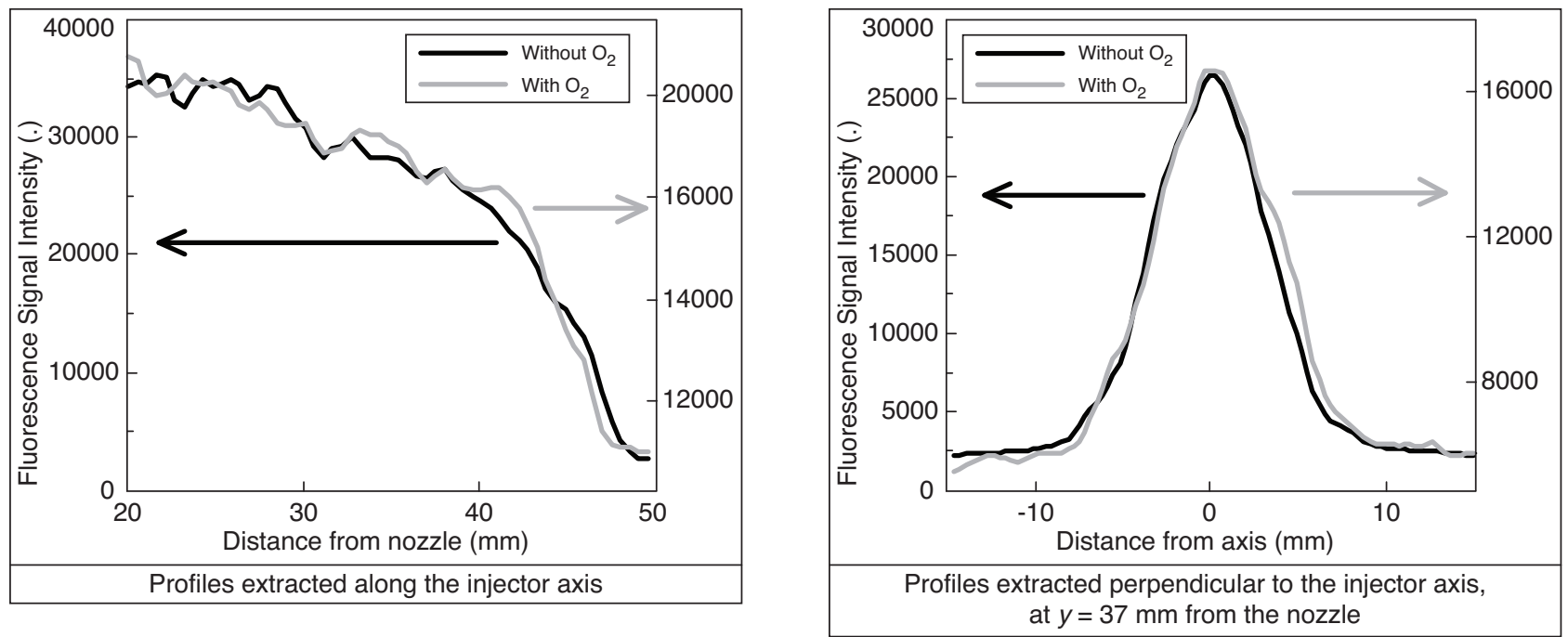

Figure 7

Comparison of profiles (extracted along and perpendicular to the injector axis) of mean averaged (10 images) of TMPD LIF, with and without oxygen. Camera A, optimized camera gains, $P_{i n j}=1200 \mathrm{bar}, t_{i}=0.8 \mathrm{~ms}$. 
the presence of oxygen compare very well, both for profiles extracted along the injector axis and perpendicular to it.

This result indicates that either the oxygen concentration is constant throughout the observed area of the jet, or that the oxygen concentration variations in the jet have no effect on TMPD fluorescence yields. Since the first conclusion is not consistent with the fact that air is entrained in the jet and is progressively mixed with the fuel [2-4], the results of Figure 7 seem to indicate that in the operating condition studied, the TMPD fluorescence is not affected by the variations of oxygen concentration. In reference [19] the authors state that the effect of quenching by oxygen on the fluorescence of TMPD saturates for oxygen concentrations higher than a threshold level. This effect could explain the result of Figure 7, but more background on the photo-physical processes of TMPD fluorescence and more experimental validation are required to draw definite conclusions.

\subsubsection{5-nonanone}

Since the previous tests showed that the application of LIEF with TMPD might be difficult in the presence of oxygen when the conditions for signal collection are not optimum, the investigation was continued in order to find a more suitable fluorescent tracer.

Figure 8 compares 5-nonanone LIF images obtained in an oxygen free environment and in the presence of oxygen. The fluorescence intensity is significantly reduced in the presence of oxygen since approximately five times less signal is collected compared to the oxygen free condition. The effect of oxygen on the fluorescence signal intensity of 5-nonanone fluorescence is

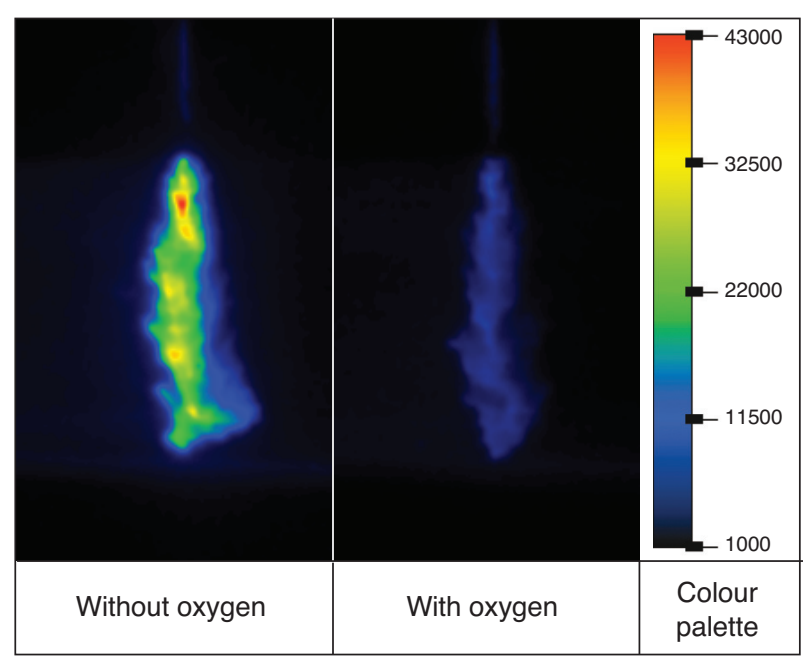

Figure 8

Comparison of individual 5-nonanone LIF images obtained with and without oxygen. Camera A, relative gain 1 , $P_{i n j}=1200 \mathrm{bar}, t_{i}=0.8 \mathrm{~ms}$, image size $40 \times 61 \mathrm{~mm}^{2}$. therefore significant in the range of operating conditions studied, as it is the case for TMPD fluorescence. This result is somehow contradictory with previous work in which it was reported that quenching effects had little influence on the fluorescence of lighter ketones [13]. However, the author found no information in the available literature of the effects of oxygen quenching on the quantum yields of 5-nonanone fluorescence.

Tracer decomposition could be a supplementary mechanism which might explain the effect of oxygen on the fluorescence signal intensity. Indeed, stability issues with 5-nonanone have been reported in previous work [14]. However, if the presence of oxygen was to increase the rate of tracer decomposition, the effect of the presence of oxygen on fluorescence intensity would be more important in the leading edge of the jet where the temperature and mixture history is favourable. Since the image of 5-nonanone fluorescence does not reveal such characteristics, it seems that tracer decomposition is not a significant issue for the range of operating conditions studied, and furthermore, that the effect of oxygen on LIF signal intensity is mainly due to the effect of quenching on 5-nonanone fluorescence quantum yields.

Although oxygen seems to have a significant effect on the quantum yield of 5-nonanone fluorescence, the detected signal remains sufficiently high to enable satisfactory visualizations in the presence of oxygen, as shown by the images of 5-nonanone fluorescence obtained in the presence of oxygen with cameras A and B, shown in Figure 9. It should be noted that the use of maximum camera gain was not necessary, even with camera B which corresponds to a set-up typical of in-cylinder engine experiments.

This result is explained by the fact that in an oxygen-free environment the acquired fluorescence signal level is very high. Indeed, the signal intensities obtained in an oxygen-free environment with TMPD and 5-nonanone can be compared from Figure 5 and Figure 8 taking into account for the relative camera gains. Approximately six times more signal is collected with 5-nonanone compared to TMPD. This result seems to indicate that the quantum yields of 5-nonanone fluorescence is higher than that of TMPD, even though an increase by a factor of two of the 5-nonanone fluorescence would be expected compared to TMPD due to the use of a higher dopant concentration. The same ratio is obtained in the presence of oxygen as shown by the comparison of Figure 6 and Figure 9, since the effect of oxygen presence on the fluorescence signal is similar for the two tracers in the range of operating conditions studied. The resulting high fluorescence intensity obtained with 5-nonanone LIF in the presence of oxygen enables satisfactory conditions for visualizations, even for the setup corresponding to engine type conditions.

This result was further improved by optimizing the collection set up for the 5-nonanone LIF. Indeed, images of Figure 9 show that the use of the $327 \mathrm{~nm}$ (FWHM $75 \mathrm{~nm}$ ) band pass 
interference filters was not optimum in terms of maximizing the LIF signal to noise ratio. The laser wavelength contribution is quite significant: the diffusion of the laser sheet light in the cell is clearly visible, and the elastically scattered light from liquid droplets within the spray is not negligible. A set of high pass $320 \mathrm{~nm}$ and low pass $400 \mathrm{~nm}$ interference filters was selected to improve signal collection efficiency and this latter filter set was used in the tracer LIF and combustion visualizations. Preliminary tests have shown that with this new set of filters, both the collected signal intensity and the signal to noise ratio were increased by a factor of two.

\subsubsection{Diverging Beam}

The evaluation of fuel mixture visualization using 5-nonanone LIF in the presence of oxygen was further investigated with a diverging beam. The objective of this supplementary test was to study the potential of 5-nonanone LIF for mixture visualization in optical engines where optical access is limited to one window (either by choice or because it is the only optical access available). Illumination at $90^{\circ}$ with a laser sheet is then not possible and global illumination is required, whilst the LIF image is retro-collected. The amount of energy available for fluorescence excitation is limited in this configuration since the laser energy is dispersed in the chamber and a significant part of the beam does not reach the jet. It is therefore useful to evaluate the potential of the 5-nonanone LIF in this configuration for engine applications.
The experimental setup was modified in order to achieve global illumination in the cell. The spherical and cylindrical lenses were replaced by a diverging spherical lens (UV-S-75D25) located such that the diverging beam cross section diameter in the centre of the cell was $4.5 \mathrm{~cm}$, corresponding to a power intensity of $10^{5} \mathrm{~W} / \mathrm{cm}^{2}$. Also, due to multiple reflections generated in the cell by this illumination, a $266 \mathrm{~nm}$ mirror was added between the cell and the camera in order to improve spectral filtering of the detected signal. Although the addition of this mirror decreases the collected signal intensity by a factor of two, a better signal to noise ratio was obtained. The set of interference filters used in the tracer LIF and combustion visualizations was not used here because it was not available at the time of the experiment.

The LIF images obtained in those conditions with camera $\mathrm{A}$ and $\mathrm{B}$ are displayed in Figure 10. The collected intensity is much lower than in the case of illumination by a laser sheet. Comparison with Figure 9 taking into account for the relative camera gains shows that signal intensity is reduced by a factor of four. However, the high signal to noise ratio of these images show that mixture visualization with 5-nonanone LIF in the presence of oxygen is possible in an optical engine type setup with global illumination.

\subsubsection{2-decanone}

The tests carried out with 2-decanone showed that the collected fluorescence signal intensity of 2-decanone is 2 to 3 times lower than that of 5-nonanone. As a result, further tests with this tracer were not performed.

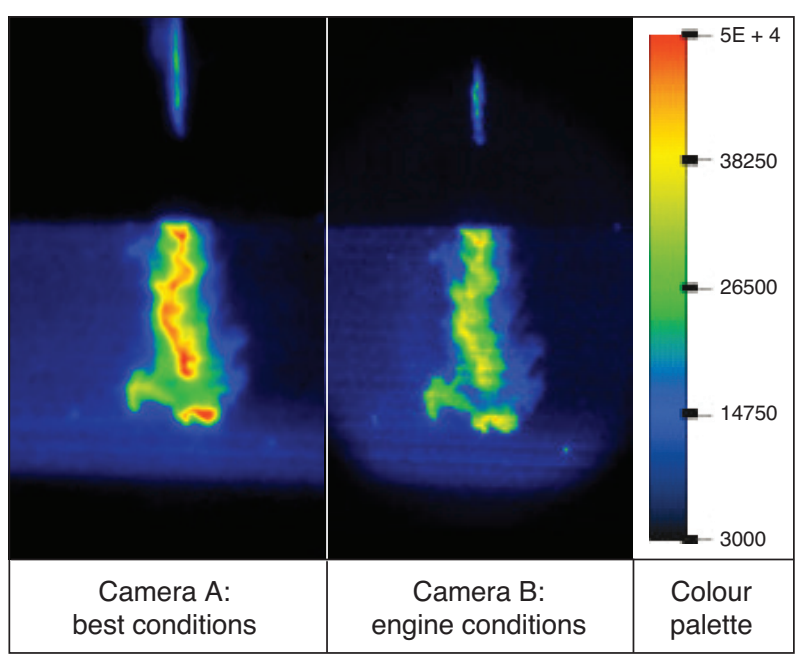

Figure 9

Comparison of individual 5-nonanone LIF images obtained in best and engine conditions (same event, same timing), with oxygen. Relative gain $4.6, P_{i n j}=1200 \mathrm{bar}, t_{i}=0.8 \mathrm{~ms}$, image size $40 \times 61 \mathrm{~mm}^{2}$.

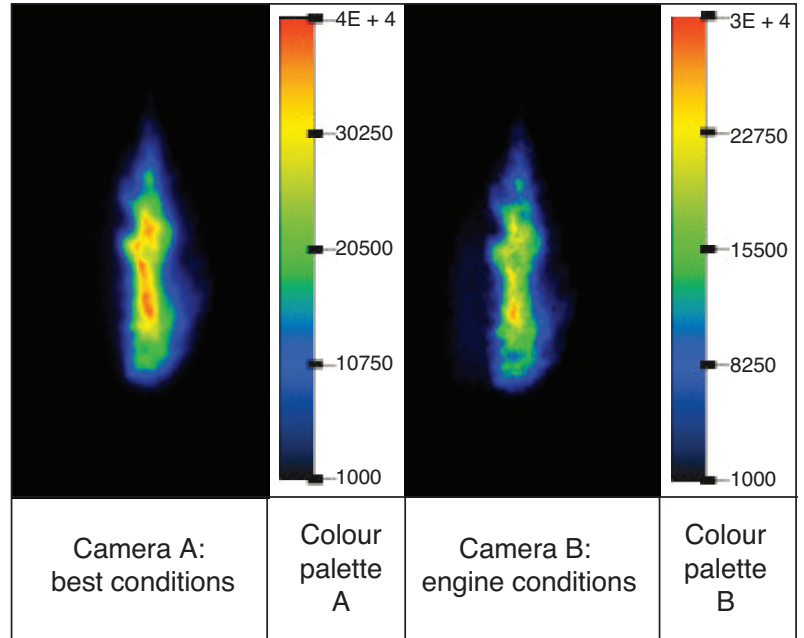

Figure 10

Comparison of individual 5-nonanone LIF images obtained in cell and engine conditions (same event, same timing), global illumination in the presence of oxygen. Relative gain 13, $P_{i n j}=1200 \mathrm{bar}, t_{i}=0.8 \mathrm{~ms}$, image size $40 \times 61 \mathrm{~mm}^{2}$. 


\subsection{High Speed Tracer LIF and Combustion Visualizations}

The tracer selection in the presence of oxygen carried out in the previous section have shown that the best results (in terms of LIF signal to noise ratio) were obtained with the 5-nonanone fluorescence. LIF with TMPD in the presence of oxygen is also possible if good visualization conditions (extensive optical access) are available. However, high speed visualizations performed here required the use of a high speed intensified camera whose collection efficiency is lower than that of the intensified single-frame CCD camera used during the tracer selection work. It is therefore very probable that the collection conditions achieved with the high speed camera would not be sufficient to enable appropriate visualizations with TMPD LIF. The 5-nonanone tracer was therefore used for high speed imaging studies of mixture distribution and combustion in the presence of oxygen.

\subsubsection{Free Jet}

A typical image sequence of 5-nonanone LIF and combustion visualizations in the free jet configuration are presented in Figure 11. The LIF image was acquired at $t_{i}=600 \mu \mathrm{s}$ after the start of injection, and the following combustion images were recorded at $20 \mu$ s intervals thereafter. The combustion image obtained at $t_{i}=620 \mu$ s displays a small luminous spot that corresponds to chemiluminescence emission and is characteristic of auto-ignition [6]. This auto-ignition region then expands progressively in the vapour plume until $t_{i}=$ $820 / 840 \mu$ s. A zone of high luminosity then appears in the centre of the vapour plume at $t_{i}=860 / 880 \mu$ s. Previous experiments [20] have shown that such bright regions appearing more than $100 \mu \mathrm{s}$ after the start of auto-ignition are due to the incandescence of soot particles. Soot therefore seems to form initially in the centre of the luminous region where auto-ignition has taken place.

For a further analysis of the temporal evolution, a second sequence of combustion images is shown in Figure 12. The first LIF image of the sequence is not displayed because it is not relevant. Indeed, this image sequence was synchronized in a similar manner to the previous one with the first image acquired at $t_{i}=600 \mu \mathrm{s}$. But in this second sequence, autoignition occurred earlier such that the chemiluminescence light emitted at the instant corresponding to LIF image acquisition was of sufficiently high intensity to dominate the LIF signal. Furthermore one would expect that any fuel/tracer present would have already been consumed by the combustion process, which would therefore reduce (or even eliminate) the LIF signal level. Since auto-ignition occurred earlier in this sequence, the interest is to present a sequence of images that provide a representative continuation of the first image sequence: the first combustion images (at $t_{i}=620 \mu \mathrm{s}$ ) of this sequence correspond to a time when auto-ignition already covers the vapour plume. It corresponds to what is

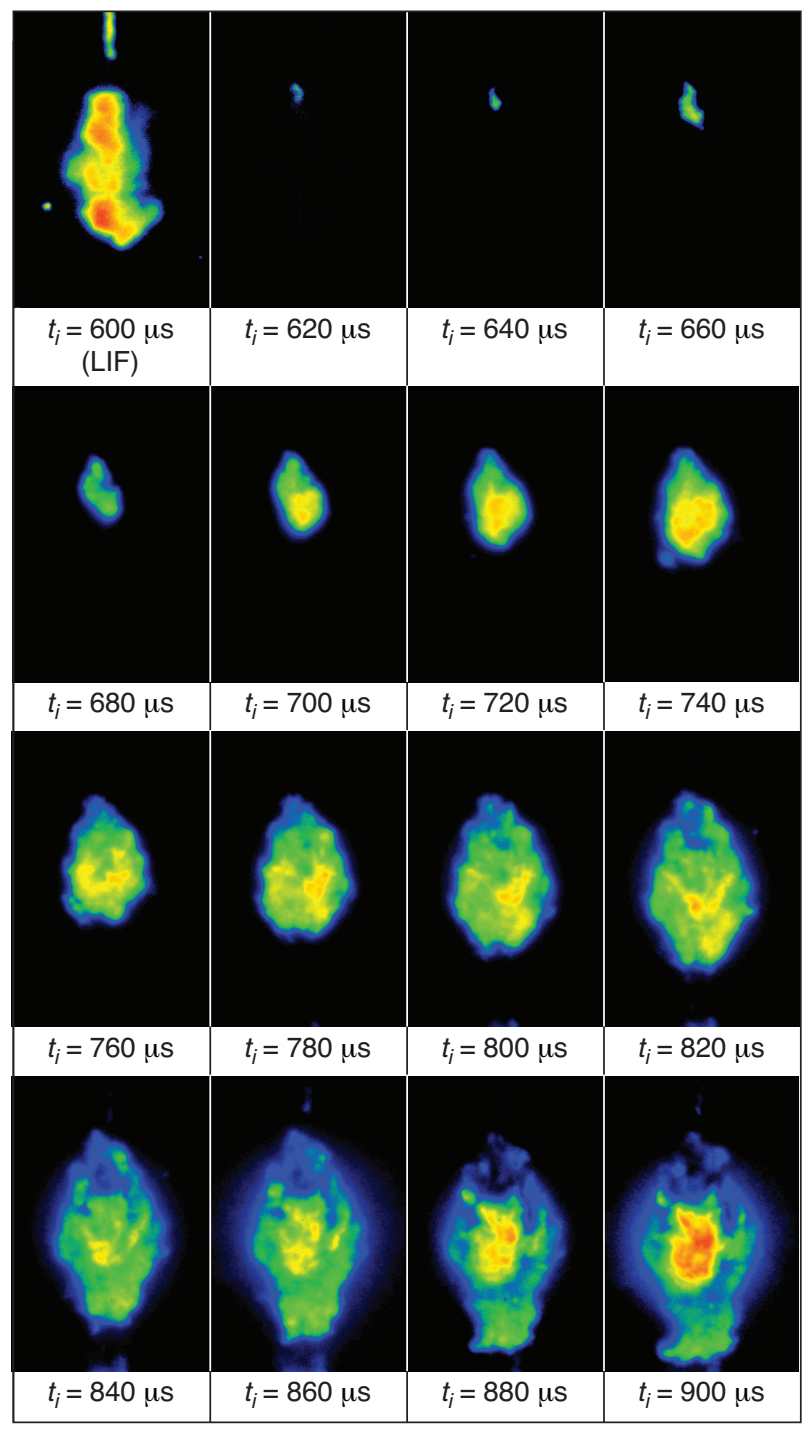

Figure 11

Time sequence of 5-nonanone LIF and direct visualizations of combustion realized in the same event with the high speed camera. $P_{i n j}=1000$ bar, image size $30 \times 45 \mathrm{~mm}$, time between images $20 \mu \mathrm{s}$.

observed in the 12th image (i.e. $t_{i}=820 \mu$ s) of the first sequence (images of Fig. 11). This second sequence of images therefore corresponds to events happening in the continuity of the sequence of Figure 11. Indeed, the same bright spot corresponding to soot formation appears in the centre of the vapour plume at approximately $700 \mu$ s. Then the bright spot progresses towards the jet tip, and the last images of the sequence reveal a mushroom-like shape, which is typical of combustion images during the diffusion flame stage [2], where the dominant light emission is due to soot incandescence. 


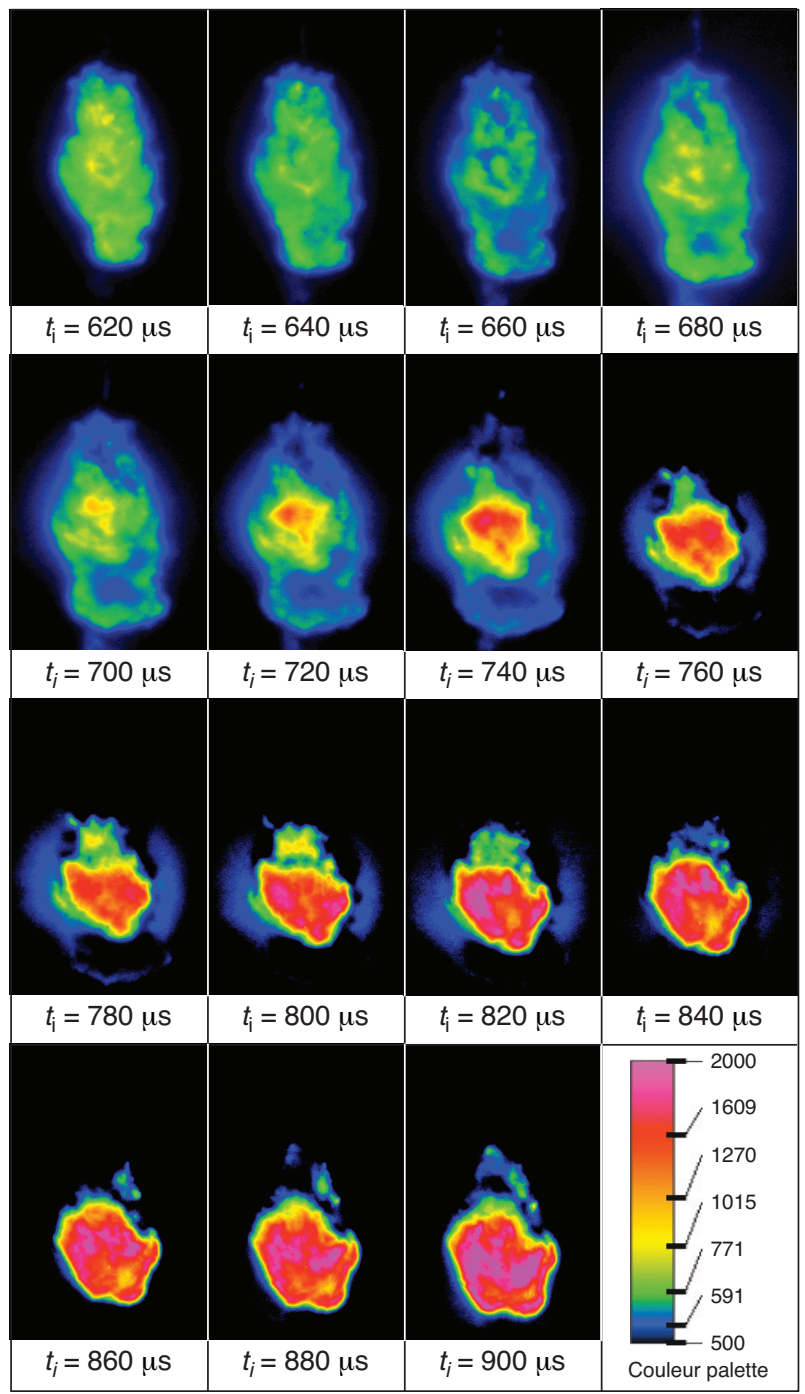

Figure 12

Time sequence of direct visualizations of combustion realized in the same event with the high speed camera. $P_{i n j}=1000 \mathrm{bar}$, image size $30 \times 45 \mathrm{~mm}$, time between images $20 \mu \mathrm{s}$.

These two image sequences therefore provide an overview of the combustion process: auto-ignition appears in localized areas before extending to the rest of the vapour plume, initial zones of soot formation then appear in the centre of this region before progressing towards the tip, and forming a mushroom-like zone.

\subsubsection{Detailed Analysis}

For a detailed analysis of the correlation between mixture distribution and auto-ignition sites, various sequences of images such as that presented in Figure 11 were processed according to the method presented earlier. The LIF and contour images resulting from this image processing is presented in Figure 13 for several image sequences carried out at an injection pressure of 1000 bar.

The spatial location of auto-ignition sites appears to be similar for the different events studied: in the upstream zone, slightly shifted from the axis of the jet. This location corresponds to the mixing zone of the jet [4].

Obtaining quantitative information on fuel concentration values at the location where auto-ignition subsequently occurs would be of great interest in order to gain information on the auto-ignition process. However this remains a challenging task for several reasons:

- Quantitative measurements of fuel concentration from 5-nonanone LIF would be required. This would need more background on the photo-physical process of 5-nonanone fluorescence, in order to accurately account for temperature, pressure and quenching effects on the quantum yields. Also, correction for laser intensity fluctuations and absorption would be necessary.

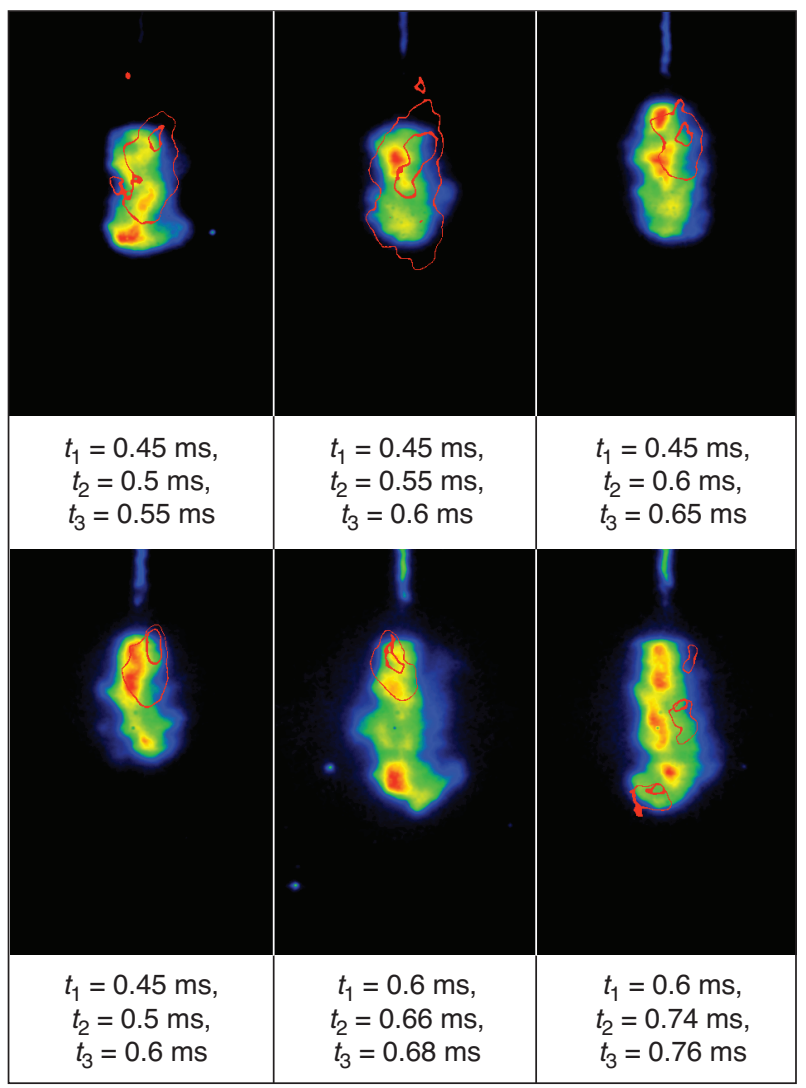

Figure 13

Processed images of LIF and contours of auto-ignition sites. $P_{i n j}=1000$ bar, image size $34 \times 54 \mathrm{~mm}^{2}$. 
- The issue of tracer decomposition would need to be studied in order to understand what the effects might be on the LIF measurements. In particular, LIF imaging is realized shortly before chemiluminescence imaging ( $50 \mu \mathrm{s}$ or more), and previous work has shown that initial autoignition precursors formed as a result of fuel decomposition appears $50 \mu$ s to $70 \mu$ s before chemiluminescence. It is therefore possible that fuel and tracer decomposition could occur at the timing where the LIF image is acquired, further complicating quantitative measurements of fuel concentration.

- During the time interval between LIF and chemiluminescence imaging, the fuel is transported by the flow so that the fuel concentration at the time and location where chemiluminescence is detected does not strictly correspond to the fuel concentration at the spatial location where the LIF image is acquired. This difference would need to be taken into account.

- LIF is a two dimensional planar laser sheet imaging technique, while direct visualization detects the natural combustion luminosity emitted over a volume (i.e. an integrated measurement), therefore corresponding to three dimensional information. The two images might therefore not correspond to the same spatial location. The application of a two dimensional diagnostic to visualize auto-ignition such as $355 \mathrm{~nm}$ LIF [7] would be more appropriate than direct combustion visualization.

It is clear from the above discussion that accurate measurements of fuel concentration at the location where autoignition subsequently occurs was not possible in the framework of the present study. However it is the author's opinion that even an approximate estimate of this value is extremely useful for the understanding of auto-ignition phenomena.

The images (in particular the second and fifth images), shown in Figure 13 reveal that the auto-ignition contours correspond closely to iso-level of fluorescence intensities which indicates a good spatial correlation between the fuel concentration distribution and location of auto-ignition sites. Furthermore, tracer decomposition does not appear to significantly affect the LIF signal since a continuous distribution of intensities is obtained in the area where auto-ignition subsequently occurs. In addition, the corresponding time delay between LIF and chemiluminescence imaging is short enough to neglect the fuel transport effects. According to these simplifications, one can therefore assume in a first approximation that the fluorescence intensities contained within the contours of the images of Figure 13 correspond to fuel concentrations at the location where auto-ignition will subsequently occur. In order to obtain a quantitative value, a calibration process is required to obtain the correspondence between pixel intensity and fuel concentration. This work was not carried out here, but it was performed in a previous study [4] under similar experimental conditions (injected quantity, ambient conditions, etc.) using a different
LIF technique. The fluorescence intensity contained within the contours of the images of Figure 13 corresponds to blue/green colours of the colour palette. The calibration method carried out in [4] reveals that this color range corresponds to fuel concentrations varying from 2 to $2.5 \mathrm{~kg} / \mathrm{m}^{3}$.

Therefore, by making a few simplifying assumptions it is possible to estimate the fuel concentration where auto-ignition sites are subsequently located to approximately 2 to $2.5 \mathrm{~kg} / \mathrm{m}^{3}$. A simple calculation of the corresponding fuel air equivalence ratio assuming that the oxygen and air concentration are equal to the ambient condition gives values of 1/1.2. The latter values are unknown but are expected to be lower in the mixing jet than in the ambient, so that the corresponding fuel air equivalence ratio based on local values is expected to be even higher.

This hypothesis, based on a number of simplifications seems to indicate that auto-ignition occurs in rich areas of the mixing zone of the jet. Further work needs to be performed in order to validate this information. However it can be noted that one of the simplifications (i.e. neglecting the effect of tracer decomposition) leads to an underestimation of the fuel concentration and it therefore has no consequence on this conclusion (i.e. if tracer decomposition was significant, the mixture would in fact be richer than estimated).

\subsubsection{Effect of Higher Injection Pressure}

Sequences of LIF and combustion images were also acquired at higher injection pressures in order to evaluate the effect of an increase in injection pressure on the correlation between mixture and auto-ignition. Figure 14 displays two sequences of 5-nonanone LIF and combustion images corresponding to an injection pressure, $P_{i n j}=1500$ bar. In general the image sequences are similar to those obtained at the lower injection pressure: auto-ignition appears in confined areas before extending to the rest of the vapour plume. However, a more detailed examination reveals that the rate of the extension process seems faster at higher pressure: the time interval between the apparition of auto-ignition sites and the extension of auto-ignition to the rest of the vapour plume is of the order of $\sim 100 \mu$ s at $P_{i n j}=1500$ bar, while the corresponding timescale is approximately $\sim 200 \mu$ s at $P_{i n j}=1000$ bar as shown by the results of Figure 11. Also, ignition occurs in multiple sites as a consequence of the faster extension of auto-ignition. The effect of increasing the injection pressure on the auto-ignition extension rate was already observed in previous work [9].

In order to confirm these tendencies, a more systematic investigation was carried out by image processing various events. Figure 15 displays LIF and contour images of several events carried out at an injection pressure of $P_{i n j}=1500$ bar. Globally the same characteristics concerning the location of auto-ignition sites are observed when compared with the results obtained at $P_{i n j}=1000$ bar: auto-ignition appears in 


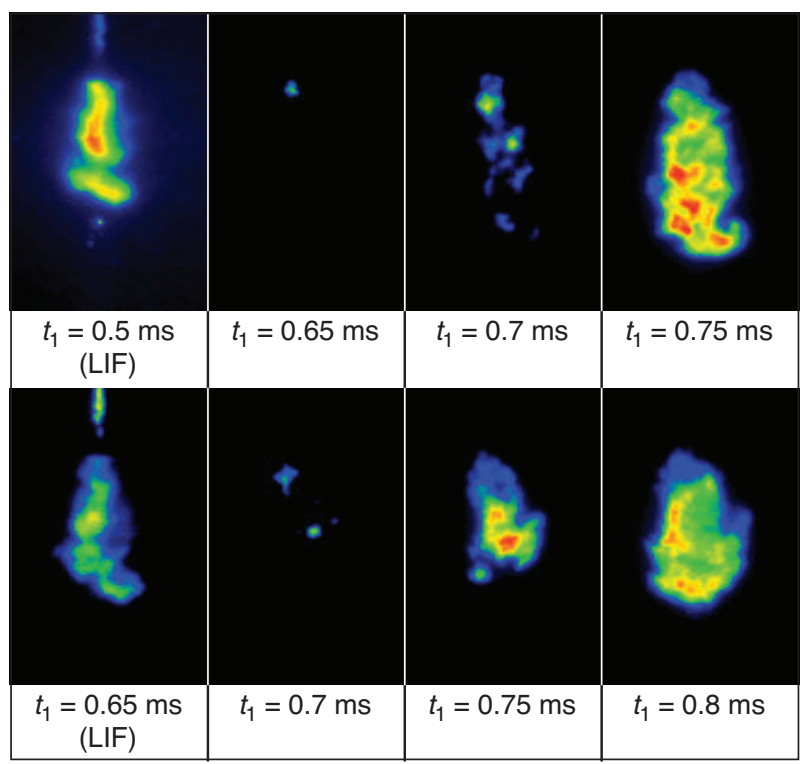

Figure 14

Two time sequences of LIF and direct visualizations of combustion realized with the high speed camera. $P_{i n j}=1500$ bar, image size $30 \times 45 \mathrm{~mm}^{2}$, time between images $50 \mu$ s.
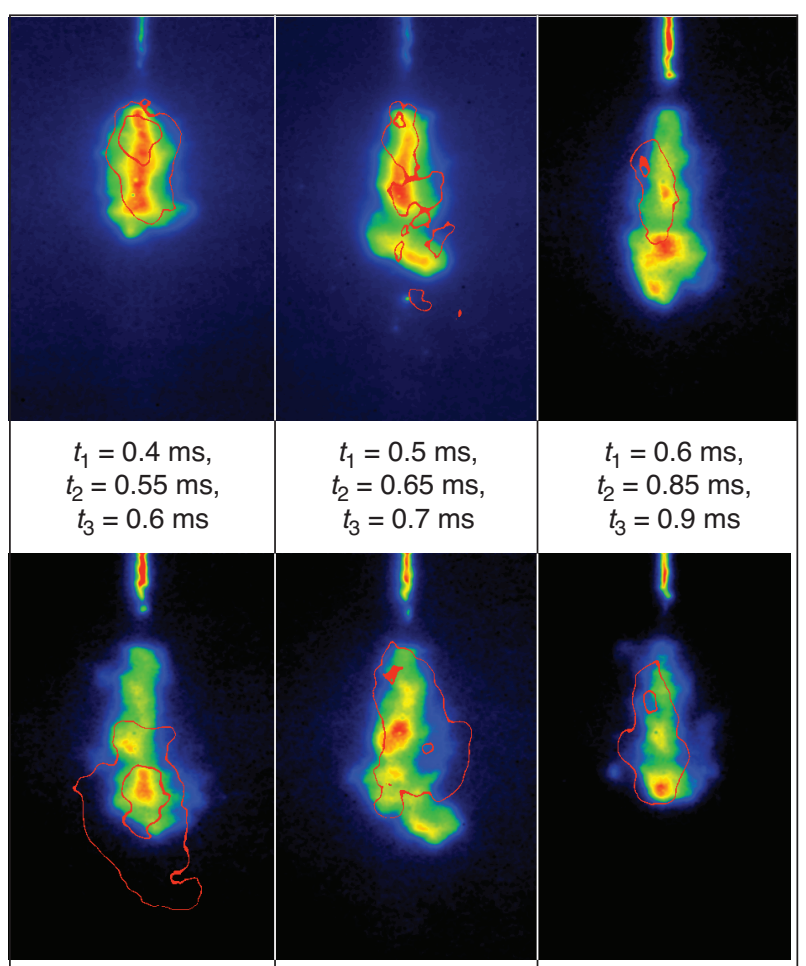

$t_{1}=0.5 \mathrm{~ms}$, $t_{2}=0.65 \mathrm{~ms}$ $t_{3}=0.7 \mathrm{~ms}$

$t_{1}=0.6 \mathrm{~ms}$ $t_{2}=0.85 \mathrm{~ms}$, $t_{3}=0.9 \mathrm{~ms}$

\begin{tabular}{c|r}
$t_{1}=0.65 \mathrm{~ms}$, & $t_{1}=0.65 \mathrm{~ms}$, \\
$t_{2}=0.95 \mathrm{~ms}$, & $t_{2}=0.7 \mathrm{~ms}$, \\
$t_{3}=1 \mathrm{~ms}$ & $t_{3}=0.75 \mathrm{~ms}$
\end{tabular}

Figure 15

Processed images of LIF and contours of auto-ignition sites. $P_{i n j}=1500$ bar, image size $34 \times 54 \mathrm{~mm}^{2}$. the upstream zone, slightly shifted from the axis of the jet. Also, the level of fluorescence intensity corresponding to auto-ignition sites is similar. However, in general the auto-ignition sites are located further downstream at $P_{i n j}=1500$ bar than at $P_{i n j}=1000$ bar. Auto-ignition is even observed at the tip of the jet for one event with an auto-ignition of $0.95 \mathrm{~ms}$. A longer distance is observed between the nozzle exit and autoignition site and this can be explained by the fact that for a similar time required for auto-ignition, the mixture travels a greater distance from the nozzle exit at higher pressure since the injection velocity increases with pressure. Also, the space between the two auto-ignition contours is generally higher at $P_{i n j}=1500$ bar than at $P_{i n j}=1000$ bar (comparing images with similar time intervals between contour detection, i.e. $50 \mu \mathrm{s})$. This result confirms that the extension process is faster at higher pressure.

\subsubsection{Wall Jet}

A study of the correlation between the fuel mixture distribution and auto-ignition was also conducted in the case of a jet impinging against a flat wall.

A sequence of LIF and combustion images in the wall jet configuration is presented in Figure 16. The LIF image is typical of a jet impinging on a flat wall [5]: the vapour plume interaction with the wall generates a vortex at the tip of the jet know as the jet wall vortex. A distinctive feature can however be observed: the 5-nonanone LIF image is significantly asymmetric. While the vortex can be easily observed on the left side of the image, it is less distinct on the right side. Since there are no reason for the jet wall interaction to create such an asymmetry, the only reasonable explanation is that the laser sheet is absorbed by the 5-nonanone. This absorption effect is especially visible in the jet wall configuration because the laser sheet path through the jet is increased in the region where the jet propagates along the wall. Indeed, this absorption effect is not significant in the free jet configuration images presented earlier, probably because the laser sheet path through the jet is not sufficient to cause significant absorption by 5-nonanone. Also, compared to jet wall LIEF images reported in Reference [5] performed using TMPD as the fuel tracer, this absorption phenomenon appears to be more significant. This is very probably due to the higher efficiency of the 5-nonanone absorption compared to TMPD.

A second sequence of LIF and combustion images obtained at a higher injection pressure ( $P_{i n j}=1400 \mathrm{bar}$ ) is presented in Figure 17. The LIF image shows a more extended vapour plume than at the lower injection pressure. This result is typical and is explained by the higher flow velocities. In this sequence, the auto-ignition site is located in the vicinity of the wall. It then extends firstly in a region restricted to the vicinity of the wall, before extending to regions more distant from the wall, upstream and downstream. Again, it seems that the presence of the wall is favourable to the propagation of auto-ignition. 


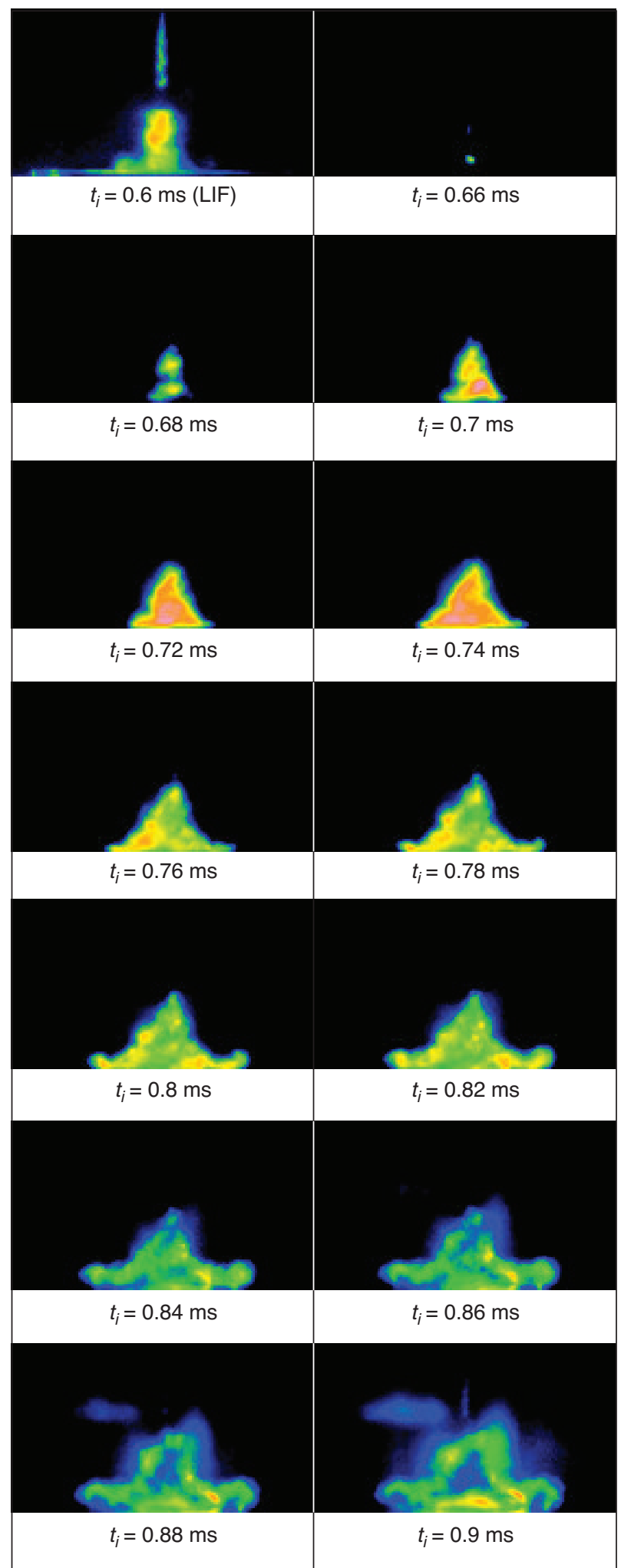

Figure 16

Time sequence of 5-nonanone LIF and direct visualizations of combustion realized in the same event with the high speed camera. $P_{i n j}=1000$ bar, $d_{n w}=3.5 \mathrm{~cm}$, image size $50 \times 31.5 \mathrm{~mm}^{2}$, time between images $20 \mu \mathrm{s}$.

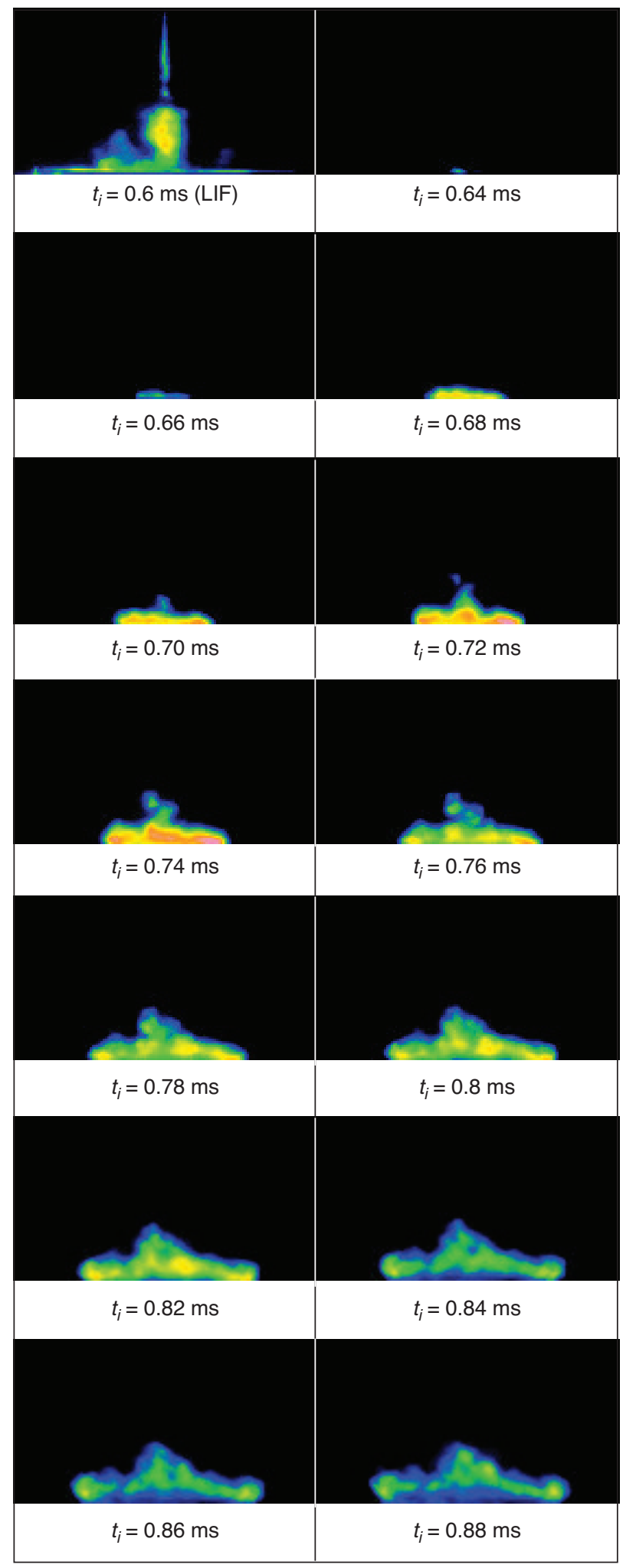

Figure 17

Time sequence of 5-nonanone LIF and direct visualizations of combustion realized in the same event with the high speed camera. $P_{i n j}=1400$ bar, $d_{n w}=3.5 \mathrm{~cm}$, image size $50 \times 31.5 \mathrm{~mm}^{2}$, time between images $20 \mu$ s. 


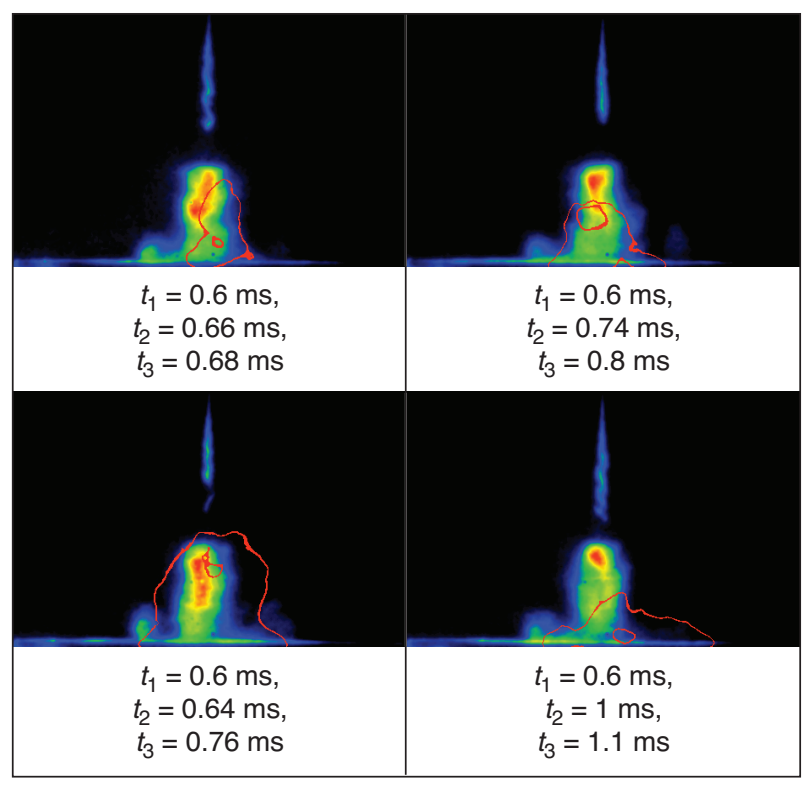

Figure 18

Processed images of LIF and contours of auto-ignition sites. $P_{i n j}=1000 \mathrm{bar}, d_{n w}=3.5 \mathrm{~cm}$, image size $50 \times 31.5 \mathrm{~mm}^{2}$.

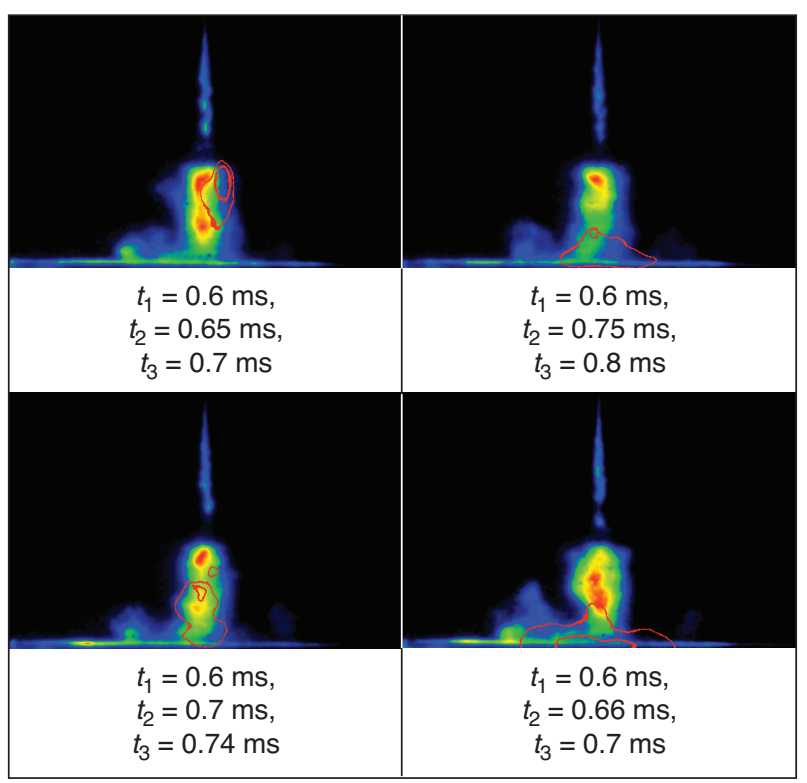

Figure 19

Processed images of LIF and contours of auto-ignition sites. $P_{i n j}=1400$ bar, $d_{n w}=3.5 \mathrm{~cm}$, image size $50 \times 31.5 \mathrm{~mm}^{2}$.

A more systematic investigation was carried out by image processing various events. Figures 18-20 display LIF and contour images of several events carried out at $P_{i n j}=1000 \mathrm{bar}$ and $d_{n w}=3.5 \mathrm{~cm}, P_{i n j}=1400$ bar and $d_{n w}=3.5 \mathrm{~cm}$, and

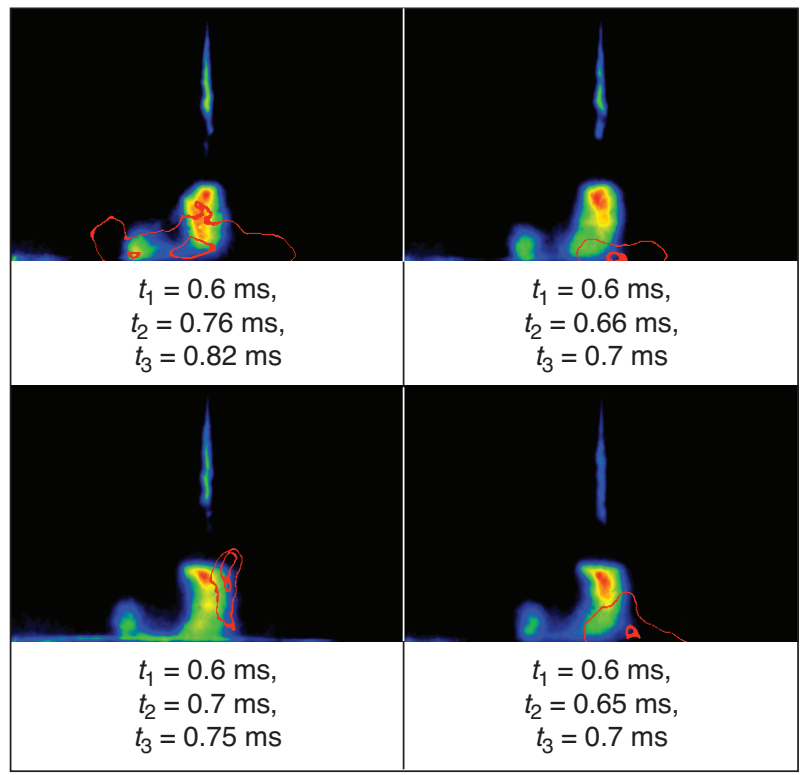

Figure 20

Processed images of LIF and contours of auto-ignition sites. $P_{i n j}=1000 \mathrm{bar}, d_{n w}=2.5 \mathrm{~cm}$, image size $50 \times 27 \mathrm{~mm}^{2}$.

$P_{i n j}=1000$ bar and $d_{n w}=2.5 \mathrm{~cm}$ respectively. Globally the same characteristics concerning the location of auto-ignition sites are observed compared to the results presented previously. Auto-ignition sites are located either in the upstream mixing zone or in the vicinity of the wall. The shape of the chemiluminescence contour that corresponds to the second timing (i.e. the more extended one) is more extended close to the wall confirming that the wall is favourable to the propagation of auto-ignition. The effect of increasing the injection pressure or decreasing the distance between the nozzle and the wall seems to increase the number of cases where autoignition is located close to the wall surface.

An explanation as to why the presence of the wall appears to be favourable to the propagation of auto-ignition is not straightforward. Indeed, the wall temperature is not known in this experiment but it would be expected to be lower than the ambient gas temperature, generating a thermal layer at the vicinity of the wall. The factor promoting auto-ignition and its propagation at the vicinity of the wall is therefore not the temperature, but more probably the mixture. Indeed, detailed visualizations [5] have shown that a characteristic mixture structure is generated during impingement in the central part of the jet, close to the wall: fuel concentration tends to increase in his zone due to the formation of a stagnation point were the jet is confined. The rich zones located at the vicinity of the wall probably promotes auto-ignition and its propagation. 
Finally, as shown by the strong asymmetry of the LIF images in the near wall region, laser sheet absorption is very significant in this configuration, leading to complete disappearance of the fluorescence signal in some parts of the jet. This absorption effect cannot be corrected for. The LIF images cannot therefore be used to obtain quantitative information, even approximate, concerning the local air fuel ratio. Only qualitative information in the zone of auto-ignition site is provided.

\section{CONCLUSION}

The correlation between the mixing and auto-ignition processes of high pressure Diesel jets was studied by simultaneous fluorescence and chemiluminescence imaging, both for free jet and flat wall impinging jet configurations.

First, a fuel visualization technique in the presence of oxygen was developed, showing that:

- despite the significant signal reduction due to quenching effects, TMPD LIF in the presence of oxygen is feasible if good conditions in terms of signal collection are provided;

- fluorescence of 5-nonanone also appears to suffer from quenching effects, but its quantum yield remains sufficiently high to enable good conditions for visualizations in the presence of oxygen;

- the high quantum yields of 5-nonanone fluorescence also enables the visualization of fuel in the presence of oxygen with global illumination and in conditions typical of an optical engine.

The 5-nonanone laser induced fluorescence technique was therefore selected and combined with high speed chemiluminescence imaging to study the correlation between mixing and auto-ignition.

In the free jet configuration it was found that:

- auto-ignition appears in localized areas before extending to the rest of the vapour plume, soot then appears in the centre of this region before forming a dense, mushroomlike, soot region at the jet tip;

- in the free jet configuration and for the parameter range studied, auto-ignition is preferentially located in rich regions of the upstream mixing zone;

- following an increase of the injection pressure the autoignition sites are shifted downstream and the extension process is faster.

For the jet wall configuration:

- in the parameter range studied, auto-ignition appears in the centre of the jet and propagates towards the periphery, at the vicinity of the wall;

- auto-ignition sites are located either in the upstream mixing zone, either in the vicinity of the wall;
- following an increase of the injection pressure or a decrease in the distance between the nozzle and the wall, auto-ignition appears to occur preferentially in zones close to the wall surface.

The combined techniques therefore provided detailed information on the correlation between the mixing and combustion processes. However, due to several uncertainties, the results were limited to semi-quantitative analysis. The use of a two dimensional diagnostic for the detection of auto-ignition sites, such as $355 \mathrm{~nm}$ LIF [6], could provide more detailed and quantitative information in future work.

\section{ACKNOWLEDGMENTS}

The work has been conducted with the expert technical assistance of Laurent Hermant. Also, the help of Julian Kashdan for improving the quality of the paper is greatly acknowledged. This work was supported by the GSM (Groupement Scientifique Moteurs: IFP, PSA and Renault).

\section{REFERENCES}

1 Espey C., Dec J.E., Litzinger T.A., Santavicca D.A. (1994) Quantitative 2-D Fuel Vapor Concentration Imaging in a Firing D.I. Diesel Engine Using Planar Laser-Induced Rayleigh Scattering, SAE 940682.

2 Bruneaux G. (2001) Liquid and Vapor Spray Structure in High Pressure Common Rail Diesel Injection, Atomization Spray. 11, 5, 533-556.

3 Siebers D.L. (1998) Liquid Phase Fuel Penetration in Diesel Sprays, SAE 980809.

4 Bruneaux G. (2005) Mixing Process in High Pressure Diesel Jets by Normalized Laser Induced Exciplex Fluorescence - Part I: Free Jet, SAE 2005-01-2100.

5 Bruneaux G. (2005) Mixing Process in High Pressure Diesel Jets by Normalized Laser Induced Exciplex Fluorescence - Part II: Wall Impinging Versus Free Jet, SAE 2005-01-2097.

6 Aizawa T., Kosaka H. (2006) Investigation of the early soot formation process in a transient spray flame via spectral measurements of laser-induced emissions, Int. J. Engine Res. 7, 93-101.

7 Bruneaux G., Augé G., Lemenand C. (2004) A Study of Combustion Structure in High Pressure Single Hole Common Rail Direct Diesel Injection Using Laser Induced Fluorescence of Radicals, The Sixth International Symposium on Diagnostics and Modeling of Combustion in Internal Combustion Engines, Comodia 2004, August 2-5, 2004, Yokohama, Japan, pp. 551-559.

8 Pickett L.M., Siebers D.L. (2006) Soot formation in diesel fuel jets near the lift-off length, Int. J. Engine Res. 7, 103-130.

9 Verhoeven D., Vanhemelryck J.L., Baritaud T. (1998) Macroscopic and Ignition Characteristics of High-Pressure Sprays of Single Component Fuels, SAE paper 981069.

10 Siebers D. (1985) Ignition Delay Characteristics of Alternative Diesel Fuels: Implications on Cetane Number, SAE paper 852102.

11 Pickett L.M., Lopez J.J. (2005) Jet-Wall Interaction Effects on Diesel Combustion and Soot Formation, SAE 2005-01-0921. 
12 Bruneaux G., Verhoeven D., Baritaud T. (1999) High Pressure Diesel Spray and Combustion Visualization in a Transparent Model Diesel Engine, SAE paper 1999-01-3648.

13 Schulz C., Sick V. (2005) Tracer-LIF diagnostics: quantitative measurement of fuel concentration, temperature and fuel/air ratio in practical combustion systems, Prog. Energ. Combust. 31, 75121.

14 Schulz C., Gronki J., Andersson S. (2004) Multi-Species LaserBased Imaging Measurements in a Diesel Spray, SAE 2004-011917.

15 Bruneaux G. (2008) Combustion Structure of Free and Wall Impinging Diesel Jets by Simultaneous Laser Induced Fluorescence of Formaldehyde, $\mathrm{PAH}$ and $\mathrm{OH}$, Int. J. Engine Res., to be published.

16 Kashdan J., Papagni J.F. (2005) LIF Imaging of Auto-ignition and Combustion in a Direct Injection Diesel-fuelled HCCI Engine, SAE paper 2005-01-3739.
17 Deschamps B., Ricordeau V., Depussay E., Mounaïm-Rousselle C. (1999) Combined Catalytic Hot Wires Probe and Fuel-AirRatio-Laser Induced-Exciplex Fluorescence Air/Fuel Ratio Measurements at the Spark Location Prior to Ignition in a Stratified GDI Engine, SAE 1999-01-3536.

18 Koban W., Koch J.D., Hanson R.K., Schulz C. (2005) Toluene LIF at elevated temperatures: Implications for fuel/air ratio measurements, Appl. Phys. B 80, 147-150.

19 Discussions with U. Leidenberger, LTTT, Universitat Bayreuth, Germany, during the poster session of the 31st International Symposium on Combustion, Heidelberg, 2006.

20 Bruneaux G., Lemenand Ch. (2001) Internal IFP Report No. 55879, February.

Final manuscript received in March 2008 Published online in July 2008 or distributed for profit or commercial advantage and that copies bear this notice and the full citation on the first page. Copyrights for components of this work owned by others than IFP must be honored. Abstracting with credit is permitted. To copy otherwise, to republish, to post on servers, or to redistribute to lists, requires prior specific permission and/or a fee: Request permission from Documentation, Institut français du pétrole, fax. +33147527078 , or revueogst@ifp.fr. 